\title{
Gene markers for grain polyphenol oxidase activity in common wheat
}

\author{
X. B. Wang - C. X. Ma · H. Q. Si • Y. Q. Qiao \\ C. Chang $\cdot$ X. F. He $\cdot$ Y. X. Xia
}

Received: 12 March 2008/Accepted: 6 September 2008/Published online: 10 October 2008

(C) The Author(s) 2008. This article is published with open access at Springerlink.com

\begin{abstract}
Polyphenol oxidase (PPO) in grain is regarded as a major factor resulting in time-dependent darkening of wheat end products, particularly for Asian noodles and steamed bread. Breeding wheat cultivars with low PPO activity using efficient and reliable markers is one of the best ways to reduce the undesirable darkening. In the present study, we developed a gene-specific marker (PPO05) for low PPO activity from the sequence AY515506. This marker detected double PCR fragments $(<750$ and $>750 \mathrm{bp}$ ) in the cultivars with low PPO activity and a single PCR fragment ( $<750 \mathrm{bp}$ ) in the cultivars with high PPO activity. Screening of this marker on 235 Chinese wheat micro-core collections showed that the double fragments were present in 113 genotypes and the single fragments in the remaining 122 genotypes. Statistic analysis revealed that the cultivars with the double fragments had significantly lower mean PPO activity than those with single fragments. Through sequence analysis and blast search in NCBI, we found that the cultivars with the double fragments contained the PPO-2Ab allele, while the cultivars with the single fragments contained the PPO-2Aa allele. The PPO-2Ab and PPO-2Da alleles were associated with the low grain PPO activity and the
\end{abstract}

X. B. Wang · C. X. Ma (凹) · H. Q. Si · Y.

Q. Qiao · C. Chang · X. F. He · Y. X. Xia

Agronomy Department, Anhui Agricultural University,

Hefei, Anhui, China

e-mail: machx@163.net
PPO-2Aa and PPO-2Db alleles associated with the high PPO activity. The genotypes carrying both PPO$2 \mathrm{Ab}$ and PPO-2Da showed the lowest PPO activity, while the genotypes carrying both PPO-2Aa and PPO-2Db showed the highest PPO activity. Comparison of PPO05 and STS01 with the STS markers PPO18 and PPO29 showed that the larger and small fragments of PPO05 were equivalent to the 876- and 685-bp fragments of PPO18, respectively, and that STS01 was the complementary marker of PPO29. Thus, the STS markers PPO05 and STS01 along with PPO18 and PPO29 are the efficient and reliable markers for the evaluation of PPO activity and can be used in wheat breeding programs to improve the quality of noodles and other end products.

Keywords Triticum aesticum .

Polyphenol oxidase $\cdot$ STS markers

\section{Introduction}

Noodles are staple food in many Asian countries. In China, over $40 \%$ of wheat is used to make various types of noodles (Liu et al. 2003; He et al. 2004). Chinese white noodles, white-salted Udon noodles, and yellow alkaline (ramen) noodles are the most popular types (Nagao 1996; Liu et al. 2003; He et al. 2004). Different types of noodles vary in firmness and texture, but all types require good brightness. White is the preferred color for Chinese white and Udon 
noodles. Time-dependent darkening is undesirable for all types of Asian noodles. Cantonese and Japanese noodles, which are sold fresh, are especially sensitive to the darkening problem. Many studies have pointed out that polyphenol oxidase (PPO) plays a major role in the time-dependent darkening of noodles and other wheat products (Baik et al. 1995; Hatcher et al. 1999; Miskelly 1996; Morris et al. 2000). PPO catalyzes the formation of quinones from phenols in the presence of molecular oxygen. The quinones react with amines and thiol groups or undergo self-polymerization to produce dark or brown products. Therefore, it is necessary for wheat breeding programs targeting for noodle products to develop cultivars with low PPO activity.

Genetic variation of PPO activity in wheat grain is observed in different genotypes, and PPO activity is also influenced by environment (Park et al. 1997; Ge et al. 2003). As PPO activity is a physiologicalbiochemical trait, it cannot be selected based on morphological characteristics. Identification and use of molecular markers linked to the major QTLs associated with PPO activity could accelerate the efficiency of selection for low PPO activity.

PPO genes have been cloned and sequenced in several plant species, including common wheat (Triticum aestivum L.) (Demeke and Morris 2002). Seven PPO genes were identified in tomato (Lycopersicon esculentum Mill.) (Thipyapong et al. 1997) and 12 isozymes of PPO were reported for wheat (Kruger 1974). Several major qualitative trait loci associated with PPO activity were identified and mapped on wheat homoeologous group 2 in various wheat populations (Mares and Campbell 2001; Raman et al. 2005). Simeone et al. (2002) mapped a gene for high PPO activity on the long arm of chromosome 2A using a set of RILs, and identified a RFLP marker, Xutv 1427-2A, highly associated with the high PPO activity. Jukanti et al. (2004) cloned six PPO genes and grouped these PPO genes into two clusters. Three PPO genes (GenBank Accession Numbers AY596268, AY596269 and AY596270) in one cluster were expressed during kernel development and might influence PPO activity in wheat flour. Sun et al. (2005) developed a STS marker PPO18, which was considered as an efficient and reliable molecular marker for wheat kernel PPO activity, based on the sequence of AY596268 located on 2A. Anderson (2004) submitted to NCBI a cDNA sequence of PPO (Accession Number AY515506), which expressed predominately in developing grain and was located on chromosome 2D (Chang et al. 2006). He et al. (2007) characterized the PPO genes on chromosomes 2A and 2D and developed two complementary dominant STS markers, PPO16 and PPO29, for the PPO-2D gene. In a previous study, we developed a STS marker (STS01), which could effectively discriminate two alleles of PPO-2D gene, from the sequence AY515506 (Wang et al. 2008).

In the present study, we have developed a PCR marker PPO05, which amplifies double fragments ( $<750$ and $>750 \mathrm{bp}$ ) in the cultivars with low PPO activity, but single PCR fragments $(<750 \mathrm{bp})$ in the cultivars with high PPO activity, from the AY515506 sequence. The marker was used to screen 235 Chinese wheat micro-core collections suitable for Changjiang and Huaihai regions to validate the correlation between the marker and grain PPO activity. Marker testing and sequence analysis revealed that the cultivars with different PPO activities contained the different alleles of the PPO genes located on chromosome 2A and 2D. STS markers PPO18 and PPO29 were also screened on the 235 Chinese wheat microcore collections to compare with PPO05 and STS01. The identification and validation of the markers and the alleles of PPO genes would facilitate the marker assisted selection (MAS) of low PPO activity for the improvement of noodle and other end product quality in wheat breeding.

\section{Materials and methods}

\section{Plant materials}

In total, 235 Chinese wheat micro-core collections, kindly provided by Professor Jia Jizeng in CAAS, were used to validate the STS markers developed in this study. Four cultivars with high PPO activity and four with low PPO activity were used to identify the markers linked to PPO activity.

Field trials and grain PPO activity assay

During the 2004-2005 and 2005-2006 crop seasons, the 235 Chinese wheat micro-core collections were grown in two locations of the experimental station of Anhui Agricultural University, located in Hefei and 
Fengyang, Anhui Province, China. The experiment was performed using randomized complete block design with two replications. Each plot contained two 2 -m rows spaced $25 \mathrm{~cm}$ apart, with 100 plants in each row.

PPO activity was measured using the spectrophotometric method reported by Anderson and Morris (2001) and Han et al. (2006) with modifications. In brief, $7.5 \mathrm{ml}$ of freshly prepared $10 \mathrm{mM} \mathrm{L}-\mathrm{DOPA}$ (3, 4-dihydroxyphenylalanine) were added to a 50-ml tube containing $50 \mathrm{mM}$ MOPS (3-( $N$-morpholino) propane sulfonic acid) buffer ( $\mathrm{pH} 6.5)$ and $0.3 \mathrm{~g}$ whole wheat meal, and the tubes were then incubated in a $37^{\circ} \mathrm{C}$ water bath and shaken with an orbital shaker at a speed of $100 \mathrm{rpm}$ for $5 \mathrm{~min}$. After incubation, the tubes were transferred into ice water to slow down the enzyme reaction and the reaction solution was immediately filtered through Whatman filter paper. Absorbance $\left(A_{475}\right)$ was measured with $0.3 \mathrm{ml}$ of the incubated solution at $475 \mathrm{~nm}$ using a Unico UV-4802H spectrophotometer. One unit of PPO activity was defined as the absorbance $\left(A_{475}\right)$ value of 1-mg sample through $1-\mathrm{cm}$ path for $1 \mathrm{~min}$ at $475 \mathrm{~nm}$. Each reaction was repeated three times.

Primer design and STS analysis

Genomic DNA was extracted from a single dried wheat seed using the method described by Kang et al. (1998). Primers (PPO05) were designed using the Primer Premier 5.0 software based on the sequence information of PPO EST AY515506 sequence, and used to screen the cultivars with low and high PPO activities. The primer sequences of PPO05 are as shown below.

\section{PPO05 Forward primer 5'-TCCGCAACTGCCAA ACGC-3'}

Reverse primer 5'-GGTCCCACTGGAGTC AAGGTC-3'

All PCR reactions were performed in a $\mathrm{P} \times 2$ thermal cycler in a total volume of $20 \mu \mathrm{l}$ containing $20 \mathrm{mM}$ of Tris- $\mathrm{HCl}$ (pH 8.4), $20 \mathrm{mM}$ of $\mathrm{KCl}$, $200 \mu \mathrm{M}$ of each dNTP, $1.5 \mathrm{mM}$ of $\mathrm{MgCl}_{2}, 10 \mathrm{nM}$ of each primer, 1.5 units of Taq polymerase, and $100 \mathrm{ng}$ of template DNA. Amplification program was $95^{\circ} \mathrm{C}$ for $5 \mathrm{~min}$, followed by 40 cycles of tough-down $\mathrm{PCR}$ at $95^{\circ} \mathrm{C}$ for $30 \mathrm{~s}, 62-50^{\circ} \mathrm{C}\left(66-54^{\circ} \mathrm{C}\right.$ for PPO29) for $30 \mathrm{~s}$ with the annealing temperature decreased by $0.3^{\circ} \mathrm{C}$ per cycle, and $72^{\circ} \mathrm{C}$ for $1 \mathrm{~min}$, with a final extension of $72^{\circ} \mathrm{C}$ for $5 \mathrm{~min}$. STS01 PCR amplifications were performed at $94^{\circ} \mathrm{C}$ for $5 \mathrm{~min}$, followed by 36 cycles of $94^{\circ} \mathrm{C}$ for $1 \mathrm{~min}, 57.5^{\circ} \mathrm{C}\left(60^{\circ} \mathrm{C}\right.$ for PPO18) for $1 \mathrm{~min}, 72^{\circ} \mathrm{C}$ for $1 \mathrm{~min}$, with a final extension of $72^{\circ} \mathrm{C}$ for $8 \mathrm{~min}$. Amplified PCR fragments were separated on a $1.5 \%$ agarose gel and stained with EB and visualized under UV light.

Sequencing of PCR products

The PCR fragments amplified from four cultivars of high PPO activity (Lumai19, Shanhan 8675, Lankao86, Enesco) and four of low PPO activity (Dixiuzao, Baimangmai, Zijiehong, Lanxizaoxiaomai) by the primer pair PPO05 were sequenced from both directions by Shanghai Sangon Biological Engineering \& Technology and Service Co., Ltd. (http://www.sangon.com). Sequence alignment was conducted using the software DNAMAN (http:// www.lynnon.com).

Statistical analysis

The average grain PPO activity of each of the 235 Chinese wheat micro-core collections was calculated from the data collected in two crop seasons and used to validate the association the STS markers with PPO activity. Analysis of variance (ANOVA) was performed with the SAS System for Windows version 9.0.

\section{Results}

Identification and validation of the STS marker PPO05

As shown in Fig. 1, clear polymorphisms were detected between the cultivars with high and low PPO activities by the marker PPO05. PPO05 amplified double PCR fragments $(<750$ and $>750 \mathrm{bp}$ ) in the four cultivars with low PPO activity, but one PCR fragment $(<750 \mathrm{bp})$ in the four cultivars with high PPO activity.

PPO05 was further validated on 235 Chinese wheat micro-core collections to investigate the association of the marker with the PPO activity. Of 235 genotypes tested, 87 genotypes with PPO activity less than 210 were always associated with the double 


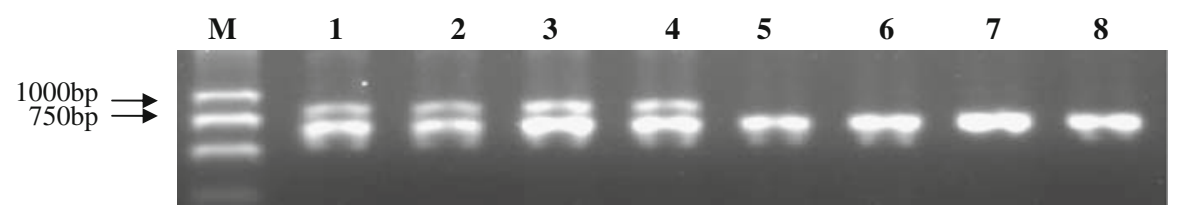

Fig. 1 PCR fragments amplified by PPO05. Lanes 1-4, cultivars with low grain PPO activity: 1 Dixiuzao (106), 2 Baimangmai (108.78), 3 Zijiehong (111.11), 4 Lanxizaoxiaomai

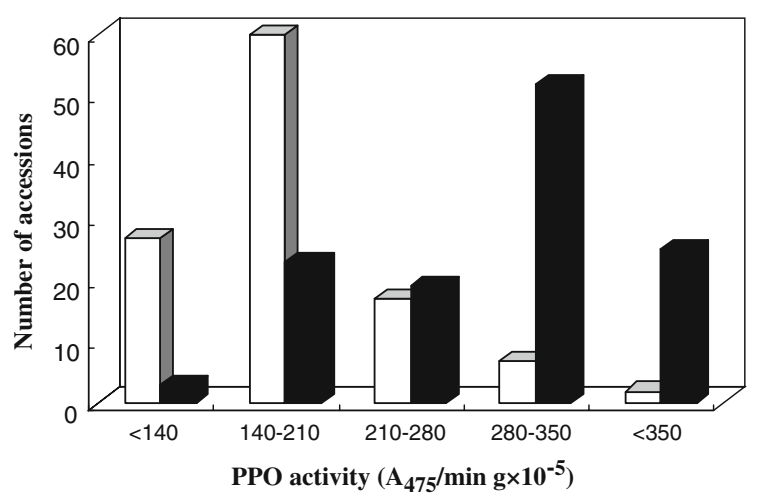

Fig. 2 Association of PPO activity with the PCR fragments amplified by PPO05 on 235 Chinese wheat micro-core collections. White columns indicate the number of accessions with the double PCR fragments; black columns indicate the number of accessions with the single PCR fragment

fragments, whereas 77 genotypes with PPO activity more than 280 were associated with the single fragments (Fig. 2). The double fragments were detected in 113 genotypes and the single fragments in the remaining 122 genotypes. Statistical analysis indicated that the average PPO activity of the cultivars with double fragments was significantly lower than that of the cultivars with single fragments (Fig. 3).

Analysis of the DNA sequences amplified by PPO05

Based on the sequence of AY515506 (162-694 bp) and annealing sites of forward and reverse primers of PPO05, a 533-bp fragment was expected to be amplified. However, the single fragments $(<750 \mathrm{bp})$ were amplified in the four cultivars with high PPO activity, while two fragments $(<750$ and $>750 \mathrm{bp})$ were amplified in the four cultivars with low PPO activity (Fig. 1). Sequence analysis indicated that the two fragments amplified by PPO05 from the four cultivars (Dixiuzao, Baimangmai, Zijiehong,
(114). Lanes 5-8, cultivars with high PPO activity: 5 Lankao86 (425.11), 6 Enesco (426.78), 7 Lumai 19 (453.22), 8 Shanhan 8675 (492.22)

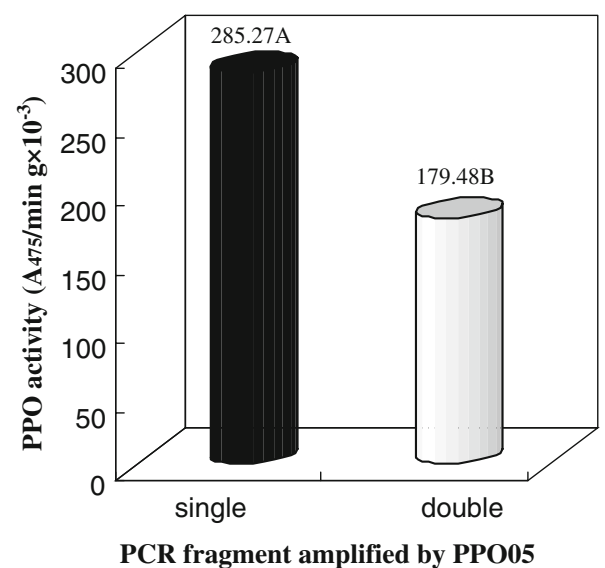

Fig. 3 Average PPO activities of the genotypes with different PCR fragments amplified by PPO05 on 235 Chinese microcore collections. Letters $A$ and $B$ on the top of the columns indicate the significant difference $(t=0.01)$ of means of PPO activities between the genotypes with single and double fragments

Lanxizaoxiaomai) with low grain PPO activity were about 636- and 826-bp, respectively. Genomic DNA sequence alignment of the four low PPO cultivars indicated that a 191-bp insertion and several SNPs were present in the larger fragment $(>750 \mathrm{bp})$ as compared to the smaller fragment $(<750 \mathrm{bp})$.

BLAST search indicated that the 636-bp (L1) sequence had $100 \%$ identity with the PPO gene PpoD1a (GenBank accession number EF070149; He et al. 2007) and was equivalent to the Ppo-D1a sequence from 271 to $906 \mathrm{bp}$, while the 826-bp (L2) sequence had $100 \%$ identity with Ppo-A1b (GenBank accession number EF070148; He et al. 2007) and was equivalent to the Ppo-A1b sequence from 291 to 1,116 bp. This indicated PPO genes on both wheat chromosome 2A and 2D were amplified simultaneously by PPO05. To reflect the chromosome locations of the PPO genes, the two PPO genes were renamed in this study as PPO-2A and PPO-2D, i.e., the PPO genes on chromosome $2 \mathrm{~A}$ and $2 \mathrm{D}$, 
respectively. Letters " $a$ " and " $b$ " were used to represent two alleles of each gene. Therefore, Ppo$\mathrm{A} 1 \mathrm{a} / \mathrm{b}$ in He et al. (2007) becomes as PPO-2Aa/b and Ppo-D1a/b as PPO-2Da/b in the rest of this article.

The fragments amplified by PPO05 from each of four cultivars (Lumai 19, Shanhan 8675, Lankao86, Enesco) with high PPO activity also contained two sequences although a single band was observed in agarose gel. The fragments amplified from Lumai 19 and Shanhan 8675 contained 635- and 636-bp sequences, respectively. BLAST search indicated that the 635-bp sequence demonstrated 100\% identity with the PPO gene PPO-2Aa (GenBank accession number EF070147) and was equivalent to the PPO-2Aa sequence from 292 to $926 \mathrm{bp}$, while the 636-bp sequence demonstrated $100 \%$ identity with the PPO gene PPO-2Da (GenBank accession number EF070149) and was equivalent to the PPO-2Da sequence from 271 to $906 \mathrm{bp}$. The fragments amplified from Lankao86 and Enesco contained 635- and 639-bp sequences and demonstrated $100 \%$ identity with the PPO-2Aa and PPO-2Db (GenBank accession number EF070150), respectively. The 635-bp sequence was equivalent to the PPO-2Aa sequence from 292 to 926 $\mathrm{bp}$ and the 639-bp sequence equivalent to the PPO-2Db sequence from 266 to $904 \mathrm{bp}$.

Genetic effects of PPO alleles on grain PPO activity

The sequence alignment and analysis revealed that the cultivars from which the double fragments were amplified by PPO05 contained the PPO-2Ab allele, while cultivars from which the single fragment was amplified contained the PPO-2Aa allele. Two alleles of the PPO gene on chromosome 2D (PPO-2D) could not be clearly discriminated through agarose gel electrophoresis, as two alleles differed only for 5 bp (a single base and a "TATA" insertion/deletion) in intron 1 . STS01 is a STS marker that was developed based on the SNP between two alleles of the PPO-2D gene (PPO-2Da and PPO-2Db) from the mRNA sequence of AY515506 in our former study. It can effectively discriminate two alleles of the PPO-2D gene (Wang et al. 2008). Therefore, this marker was used to discriminate the two alleles of the PPO-2D gene.

Analysis of the association between the alleles of PPO gene and PPO activity in the 235 Chinese microcollections showed that the " $b$ " allele of PPO-2A, i.e., PPO- $2 \mathrm{Ab}$, and "a" allele of PPO-2D, i.e., PPO$2 \mathrm{Da}$, were usually associated with low grain PPO activity (Table 1), while the "a" allele of PPO-2A and " $b$ " allele of PPO-2D were associated with high grain PPO activity. These results were consistent with those of $\mathrm{He}$ et al. (2007). Wheat cultivars with the genotypes of both PPO-2Aa and PPO-2Db showed the highest PPO activity (319.69), while the cultivars carrying both PPO-2Ab and PPO-2Da the lowest (176.09), and with either PPO-2Ab/-2Db or PPO$2 \mathrm{Aa} /-2 \mathrm{Da}$ the intermediate with the PPO activity level of 200 and 271.97, respectively (Table 1).

PPO18 and PPO29 were two STS markers for PPO genes on chromosome 2A and 2D respectively (Sun et al. 2005; He et al. 2007). In this study, these two markers were also screened on the 235 Chinese micro-core collections that were tested with PPO05 and STS01. As shown in Figs. 1 and 4, PPO18 amplified an 876-bp fragment in the cultivars that double PCR fragments were amplified with PPO05. In the cultivars that the single PCR fragment was amplified with PPO05, PPO18 amplified a 685-bp fragment. Thus, the larger and smaller fragments of
Table 1 Association between the PPO genotypes and PPO activity in 235 Chinese wheat micro-core collections tested with PPO05 and STS01

a Different letters in this column indicate significant differences $(P<0.01$; Fisher's protected LSD) among different genotypes

\begin{tabular}{lllll}
\hline $\begin{array}{l}\text { Markers or marker } \\
\text { combination }\end{array}$ & Alleles & $\begin{array}{l}\text { Number of } \\
\text { accession }\end{array}$ & $\begin{array}{l}\text { Mean PPO } \\
\text { activity }^{\mathrm{a}}\end{array}$ & Range \\
\hline PPO05 (PPO18) & PPO-2Aa & 122 & $285 \mathrm{~A}$ & $119-492$ \\
& PPO-2Ab & 113 & $179 \mathrm{~B}$ & $106-382$ \\
STS01 (PPO29) & PPO-2Da & 184 & $221 \mathrm{~A}$ & $106-492$ \\
& PPO-2Db & 51 & $281 \mathrm{~B}$ & $126-426$ \\
PPO05 \& STS01 & PPO-2Ab/2Da & 97 & $176 \mathrm{~A}$ & $106-382$ \\
& PPO-2Ab/2Db & 16 & $200 \mathrm{~B}$ & $126-304$ \\
& PPO-2Aa/2Da & 88 & $272 \mathrm{C}$ & $119-492$ \\
& PPO-2Aa/2Db & 34 & $320 \mathrm{D}$ & $145-426$ \\
\hline
\end{tabular}




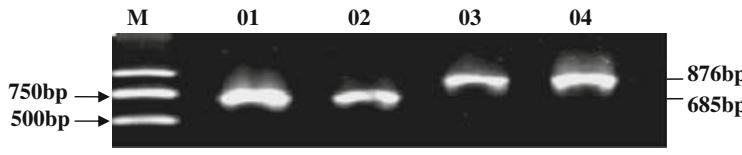

Fig. 4 Electrophoresis of PCR fragments amplified by PPO18 demonstrating two alleles (2Aa and 2Ab) of the PPO-2A gene. $M$ DNA marker, 1 Padus (2Aa), 2 Dahongpi (2Aa), 3 Dixiuzao (2Ab), 4 Lanxizaoxiaomai (2Ab)

PPO05 were equivalent to the 876- and 685-bp fragments of PPO18, respectively. It was found that STS01 was the complementary marker of PPO29 (Table 1, Figs. 5 and 6).

In addition, it was notable that number of the accessions having the PPO-2Da allele (184) was three times more than the accessions having the PPO-2Db allele (51), while the number of the accessions having either PPO-2Aa allele (122) or PPO-2Ab allele (113) was not significantly different among the 235 Chinese micro-core collections tested (Table 1).

\section{Discussion}

The introns of the PPO genes on chromosome 2A and $2 \mathrm{D}$

In this study, PPO genes (PPO-2A and PPO-2D), including the first intron (intron 1), on wheat chromosomes 2A and 2D were amplified simultaneously by the primer pair PPO05. Through sequence alignment and blast search, we found that intron 1 has a typical $5^{\prime}$-GT and $3^{\prime}$-AG boundary, which was in agreement with previous reports (Chang et al. 2006; He et al. 2007). He et al. (2007) found that both PPO$2 \mathrm{~A}$ and $2 \mathrm{D}$ had two introns and that the second intron (intron 2) was bordered by GC/AG, instead of the

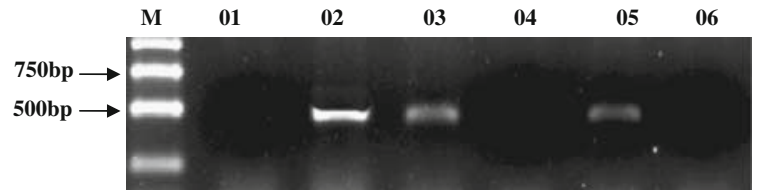

Fig. 6 Electrophoresis of PCR fragments amplified with PPO29. $M$ DNA marker, 1 Dixiuzao (2Da), 2 Dahongpi (2Db), 3 Padus (2Db), 4 Lanxizaoxiaomai (2Da), 5 Enesco (2Db), 6 Baimangmai (2Da)

typical consensus structure of GT-AG. In the present study, by analyzing the eight introns of four alleles of the two PPO genes (EF070147-EF070150), the 5' and $3^{\prime}$ exon-intron junctions were all found to be $5^{\prime}$-CAG (Gln)/G and 3'-CAG. Introns can influence gene transcription by alternative splicing (Fedorova and Fedorov 2003), and the $5^{\prime}$ and $3^{\prime}$ exon/intron junctions might control the splicing efficiency (Masao et al. 1994). Thus, it needs to be further studied whether the same $5^{\prime}$ and $3^{\prime}$ exon-intron junctions of the PPO genes on chromosome $2 \mathrm{~A}$ and $2 \mathrm{D}$ have the same splicing efficiency.

The homology comparison of PPO gene sequences

PPO05 primers were designed from the sequence AY515506, which was positioned on chromosome 2DL using nulli-tetrasomic lines in our former study. Interestingly, the double PCR fragments were amplified in four cultivars with low PPO activity while only one PCR fragment was amplified in four cultivars with high PPO activity. The sequence analysis and blast search in NCBI web site showed that PPO genes on wheat chromosome 2A and 2D were amplified by the PPO05 primers simultaneously. Sequence alignment showed that the primer sequences of PPO05

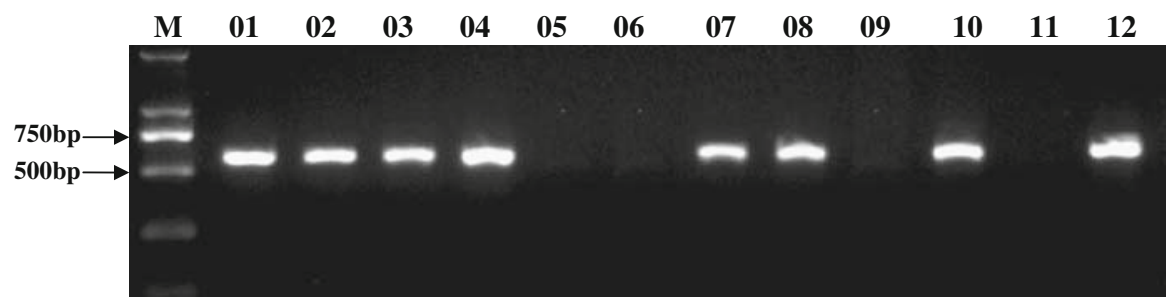

Fig. 5 Electrophoresis of PCR fragments amplified with STS01 demonstrating two alleles of PPO-2D gene (2Da/b). M DNA marker, 1 Dixiuzao (2Da), 2 Baimangmai (2Da), 3 Zijiehong
(2Da), 4 Lanxizaoxiaomai (2Da), 5 Dahongpi (2Db), 6 Padus (2Db), 7 Afu (2Da), 8 Zhahong (2Da), 9 Lankao86 (2Db), 10 Sumai 3(2Da), 11 Enesco (2Db), 12 Liuzhutou (2Da) 
Table 2 Homology of four alleles of wheat PPO genes and the AY515506 sequence

\begin{tabular}{lccccc}
\hline Allele & $\begin{array}{l}\text { PPO- } \\
2 \mathrm{Aa}\end{array}$ & $\begin{array}{l}\text { PPO- } \\
2 \mathrm{Ab}\end{array}$ & $\begin{array}{l}\text { PPO- } \\
2 \mathrm{Da}\end{array}$ & $\begin{array}{l}\text { PPO- } \\
\mathrm{Db}\end{array}$ & AY515506 \\
\hline PPO-2Aa & 100 & - & - & - & - \\
PPO-2Ab & 99.6 & 100 & - & - & - \\
PPO-2Da & 95.3 & 95.1 & 100 & - & - \\
PPO-2Db & 95.3 & 95.2 & 95.3 & 100 & - \\
AY515506 & 68.8 & 62.9 & 69.3 & 65.4 & 100 \\
\hline
\end{tabular}

were conserved in all four alleles of the PPO genes. As shown in Table 2, the sequences of PPO-2Aa and PPO-2Ab shared $99.6 \%$ of sequence identity, while PPO-2Da and PPO-2Db shared 95.3\%. He et al. (2007) reported $95 \%$ of sequence identity between the PPO-2A and PPO-2D gene. The sequence of AY515506 exhibited more than $62 \%$ of sequence identity with PPO-2A or PPO-2D, even though AY515506 was a cDNA sequence and did not include introns. The high homology between these sequences made it possible to amplify and clone the PPO genes located on different chromosomes simultaneously using primers designed from the conservative regions of the different genes.

\section{Gene markers for wheat PPO activity}

Polyphenol oxidases (PPOs) present in mature wheat kernels are implicated in the undesirable brown discoloration for noodles (Hatcher et al. 1999; Anderson and Morris 2003). Breeding wheat cultivars with low PPO activity is one of the best approaches to reduce the undesirable darkening. Molecular markers could greatly improve selection efficiency in breeding programs. In the last several years, a large number of wheat EST sequences were deposited by different laboratories, which facilitated the development of gene based molecular markers for PPO activity. Sun et al. (2005) developed a STS marker PPO18 based on the sequence AY596268, which was mapped on chromosome 2AL. He et al. (2007) cloned the fulllength sequences of wheat $\mathrm{PPO}$ genes on chromosome 2A and 2D based on the sequences of AY515506, AY596268 and CD908212, and developed two complementary dominant functional markers, PPO16 and PPO29, based on the DNA sequences of a PPO gene on chromosome 2D. The marker PPO29 was also found to be complementary to STS01 (Wang et al.
2008), which could discriminate two alleles of the PPO-2D gene. In the present study, we developed a PPO gene-specific marker, PPO05, based on a cDNA sequence AY515506. This marker detected two fragments in the cultivars with low PPO activity and single fragments in the cultivars with high PPO activity and could be used to efficiently select the favorable alleles of the PPO genes for low PPO activity in wheat breeding. It was found that PPO05 was similar to PPO18 (Sun et al. 2005), and could effectively discriminate two alleles of PPO-2A genes. Our study showed that the PPO-2Ab and PPO-2Da alleles were associated with low PPO activity, while the PPO-2Aa and PPO-2Db associated with high PPO activity. This result provides new insight that both alleles of PPO-2Ab and PPO-2Da should be selected for breeding new wheat varieties with low PPO activity. PPO05, along with all other gene-based markers developed in the previous studies, can be efficiently used to identify genotypes with low PPO activity in wheat breeding.

Acknowledgements This work was supported by grants from National Key Technologies R and D Program (2006BAD01A02) and International Collaboration Project from Ministry of Agriculture (2006-G2b).

Open Access This article is distributed under the terms of the Creative Commons Attribution Noncommercial License which permits any noncommercial use, distribution, and reproduction in any medium, provided the original author(s) and source are credited.

\section{References}

Anderson JV (2004) Triticum aestivum polyphenol oxidase (PPO) mRNA: GenBank Accession Number AY515506. http://www.ncbi.nlm.nih.gov/

Anderson JV, Morris CF (2001) An improved whole-seed assay for screening wheat germplasm for polyphenol oxidase activity. Crop Sci 41:1697-1705

Anderson JV, Morris CF (2003) Purification and analysis of wheat grain polyphenol oxidase (PPO) protein. Cereal Chem 80:135-143. doi:10.1094/CCHEM.2003.80.2.135

Baik BK, Czuchajowska Z, Pomeranz Y, Pomeranz Y (1995) Discoloration of dough for oriental noodles. Cereal Chem 72:198-205

Chang C, Zhang HP, Xu J, You MS, Li BY, Liu GT (2006) Variation in two PPO genes associated with polyphenol oxidase activity in seeds of common wheat. Euphytica 154:181-193. doi:10.1007/s10681-006-9285-2

Demeke T, Morris CF (2002) Molecular characterization of wheat polyphenol oxidase (PPO). Theor Appl Genet 104:813-818. doi:10.1007/s00122-001-0847-3 
Fedorova L, Fedorov A (2003) Introns in gene evolution. Genetica 118:123-131. doi:10.1023/A:1024145407467

Ge XX, He ZH, Yang J, Zhang QJ (2003) Polyphenol oxidase activities of Chinese winter wheat cultivars and correlations with quality characteristics. Acta Agron Sin 29:481-485

Han J, Ma CX, Si HQ, Han LD, Chen J (2006) The genetic analysis and variation of PPO activity in the early generation of Yangmai $158 \times$ Huaimai 18. J Triticeae Crops 26(5):38-41

Hatcher DW, Symons SJ, Kruger JE (1999) Measurement of time-dependent appearance of discolored sports in alkaline noodles by image analysis. Cereal Chem 76:189-194. doi:10.1094/CCHEM.1999.76.2.189

He ZH, Yang J, Zhang Y, Quail KJ, Pena RJ (2004) Pan bread and dry white Chinese noodle quality in Chinese winter wheat. Euphytica 139:257-267. doi:10.1007/s10681-0043283-Z

He XY, He ZH, Zhang LP, Sun DJ, Morris CF, Fuerst EP et al (2007) Allelic variation of polyphenol oxidase (PPO) genes located on chromosome 2A and 2D and development of functional markers for the PPO genes in common wheat. Theor Appl Genet 115:47-58. doi:10.1007/s00122007-0539-8

Jukanti AK, Bruckner PL, Fischer AM (2004) Evaluation of wheat polyphenol oxidase genes. Cereal Chem 81:481-485. doi:10.1094/CCHEM.2004.81.4.481

Kang HW, Cho YG, Yoon UH (1998) A rapid DNA extraction method for RFLP and PCR analysis from a single dry seed. Plant Mol Biol Rep 16:1-9. doi:10.1023/A:100741 8606098

Kruger JE (1974) Changes in the polyphenol oxidases of wheat during kernel growth and maturation. Cereal Chem 53:201-213

Liu JJ, He ZH, Zhao ZD, Pena RJ, Rajaram S (2003) Wheat quality traits and quality parameters of cooked dry white Chinese noodles. Euphytica 131:147-154. doi:10.1023/ A: 1023972032592

Mares DJ, Campbell AW (2001) Mapping components of flour and noodle colour in Australian wheat. Aust J Agric Res 52:1297-1309. doi:10.1071/AR01048
Masao K, Tsutomu N, Yukinoba N, Chikashi S (1994) Meiosis-dependent mRNA splicing of the fission yeast schizosaccharomyces pombe mes1+ gene. Curr Genet 25:497-503. doi:10.1007/BF00351668

Miskelly DM (1996) The use of alkali for noodle processing. In: Kruger JE et al (eds) Pasta and noodle technology. American Association of Cereal Chemists, Inc., St. Paul, pp 227-272

Morris CF, Jeffers HC, Engle DA (2000) Effect of processing, formula and measurement variables on alkaline noodle color-toward an optimized laboratory system. Cereal Chem 77:77-85. doi:10.1094/CCHEM.2000.77.1.77

Nagao S (1996) Processing technology of noodle products in Japan. In: Kruger JE (ed) Pasta and noodle technology. American Assoc., Cereal Chemists, St. Paul, pp 169-194

Park WJ, Shelton DR, Peterson CJ, Martin TJ, Kachman SD, Wehling RL (1997) Variation in polyphenol oxidase activity and quality characteristics among hard white wheat and hard red winter wheat samples. Cereal Chem 74:7-11. doi:10.1094/CCHEM.1997.74.1.7

Raman RH, Raman H, Johnstone K, Lisle C, Smith A, Martin P et al (2005) Genetic and in silico comparative mapping of the polyphenol oxidase gene in bread wheat (Triticum aestivum L.). Funct Integr Genomics 5:185-200. doi: 10.1007/s10142-005-0144-3

Simeone R, Pasqualone A, Clodoveo ML, Blanco A (2002) Genetic mapping of polyphenol oxidase in tetraploid wheat. Cell Mol Biol Lett 7:763-769

Sun DJ, He ZH, Xia XC, Zhang LP, Morris CF, Appels R et al (2005) A novel STS marker for polyphenol oxidase activity in bread wheat. Mol Breed 16:209-218. doi: 10.1007/s11032-005-6618-0

Thipyapong P, Joel DM, Steffens JC (1997) Differential expression and turnover of the tomato polyphenol oxidase gene family during vegetative and reproductive development. Plant Physiol 113:707-718

Wang XB, Ma CX, He KQ, Si HQ, Zhang YL (2008) Development and application of a STS marker for grain PPO gene located on chromosome 2D in common Wheat. Scientia Agric Sin 39(6):1583-1590 